\title{
Do Alzheimer's Disease Risk Gene Products Actually Act in Microglia?
}

\author{
Sadayuki Hashioka ${ }^{1 *}$, Ken Inoue ${ }^{2}$, Haruo Takeshita ${ }^{3}$ and Masatoshi Inagaki ${ }^{1}$ \\ ${ }^{1}$ Department of Psychiatry, Faculty of Medicine, Shimane University, Izumo, Japan, ${ }^{2}$ Research and Education Faculty, \\ Medical Sciences Cluster, Health Service Center, Kochi University, Kochi, Japan, ${ }^{3}$ Department of Legal Medicine, Faculty of \\ Medicine, Shimane University, Izumo, Japan
}

Keywords: apolipoprotein E (APOE), triggering receptor expressed on myeloid cell 2 (TREM2), CD33, diseaseassociated microglia, monocytes, Alzheimer's disease

\section{INTRODUCTION}

The strongest genetic risk factor for late-onset Alzheimer's disease $(\mathrm{AD})$ is allele $\varepsilon 4$ of the apolipoprotein E (ApoE) gene, which was discovered in 1993 (Corder et al., 1993). In around 2010, human genome-wide association studies (GWASs) of late-onset AD cases identified novel AD risk genes, the majority of which are considered to be expressed selectively or highly in microglia (Hansen et al., 2018). These include the triggering receptor expressed on myeloid cell 2 (TREM2) (Jonsson et al., 2013), complement receptor 1 (CR1) (Lambert et al., 2009), ATP-binding cassette sub-family A membrane 7 (ABCA7) (Hollingworth et al., 2011), and CD33 (Hollingworth et al., 2011). It is of note that the strength of the effect of the TREM2 variant (odds ratio of 2.362.71) is similar to that of ApoE $\varepsilon 4$ (odds ratio of 3.08) (Villegas-Llerena et al., 2016). Of these genes, ApoE, TREM2, and CD33 have been identified as key genes involved in the intermediate state of disease-associated microglia (DAM, also referred to as microglial neurodegenerative phenotype) by most recent single-cell RNA sequencing studies of microglia from AD-transgenic (Tg) mice (Keren-Shaul et al., 2017; Krasemann et al., 2017). Specifically, ApoE and TREM2 are up-regulated while CD33 is down-regulated during DAM activation (Keren-Shaul et al., 2017; Krasemann et al., 2017). Furthermore, the DAM signature is dependent on the TREM2-ApoE pathway in AD-Tg mice (Krasemann et al., 2017). Immunohistochemistry on human AD brain tissue has shown that even though DAM-like microglia (labeled with only one type of DAM marker) are located in close proximity to $\mathrm{A} \beta$ plaques, they are not found in non-plaque areas (Keren-Shaul et al., 2017; Krasemann et al., 2017). These genetic and immunohistochemistry findings imply that the functional changes of ApoE, TREM2, and CD33 caused by the variants of GWAS-identified AD risk genes, which overlap with DAM-associated genes, alter microglial functions and consequently play important roles in AD pathogenesis. Nevertheless, it should be taken into account that the protein expression of these molecules in human brains is fundamentally different from that in rodent brains.

\section{TREM2 EXPRESSION IN MICROGLIA}

A number of animal studies have demonstrated microglial immunoreactivity for TREM2 in the rodent brain (Thrash et al., 2009; Kawabori et al., 2015). In the human brain, however, there has been controversy about the microglial expression of TREM2. Even though human microglia isolated from $\mathrm{AD}$ brains show up-regulated expression of TREM2 mRNA (Gosselin et al., 2017), this increase cannot be readily confirmed at the protein level. Using the same polyclonal anti-TREM2 antibody (Sigma HPA010917), two independent immunohistochemical studies on 312 postmortem cases, including 92 $\mathrm{AD}$ cases, have shown that human microglia do not express the TREM2 protein (Satoh et al., 2013; Fahrenhold et al., 2018). These studies have also demonstrated that the TREM2-immunoreactive cells are recruited monocytes in brain blood vessels, not microglia 
or perivascular macrophages (Satoh et al., 2013; Fahrenhold et al., 2018). On the other hand, a study on 33 cases, including $11 \mathrm{AD}$ cases, reported TREM2-immunoreactive microglia associated with plaques; this study employed a different polyclonal antiTREM2 antibody (R\&D AF1828) (Lue et al., 2015). Although we have no convincing reason to explain this inconsistency in microglial immunoreactivity, the HPA010917 antibody appears to be more reliable than the AF1828 antibody, based on immunoreacting ability. Whereas AF1828 can recognize human recombinant TREM2 in immunoblotting, it is not able to label monocytes, macrophages, dendritic cells, or osteoclasts in human spleen and bone marrow (Satoh et al., 2013). The discrepancy in the human microglial expression of TREM2 between mRNA levels and protein levels could be attributed to the possibility that human TREM2 protein in microglia is post-translationally modified. For example, HPA010917 may not bind to a protein modified by glycosylation. Human TREM2 has been demonstrated to undergo glycosylation (Park et al., 2015), even though it is not known whether this kind of post-translational modification changes the TREM2 antigenicity. Another possibility is that the antibody recognizes human TREM2, but since the protein is soluble in humans, it is no longer detected by immunohistochemical methods. Soluble TREM2 resulting from ectodomain shedding has been detected in human cerebrospinal fluid by the enzyme-linked immunosorbent assay (Suarez-Calvet et al., 2019). The immunohistochemical finding that human TREM2-immunoreactive cells are largely restricted to blood circulation, not to the brain, implies that an altered immune response in the periphery may play a role in the development and progression of $\mathrm{AD}$.

\section{APOE EXPRESSION IN MICROGLIA}

ApoE is believed to be derived mainly from astrocytes in the human brain (Murakami et al., 1988). In normal human brains, ApoE is predominantly expressed by astrocytes at both mRNA (Hansen et al., 2018) and protein (Murakami et al., 1988) levels. Microglial ApoE expression is induced in AD model mice (Hansen et al., 2018). However, in human AD brains, only a portion of microglia show the immunoreactivity for ApoE (Uchihara et al., 1995), while microglial expression of the ApoE gene is up-regulated (Mathys et al., 2019). Such inconsistency in ApoE expression by $\mathrm{AD}$ microglia may stem from post-translational modification of the apolipoprotein. ApoE has recently been shown to bind to human TREM2 with a specific high affinity (Atagi et al., 2015). However, the exact relationship between ApoE and microglia in the human brain remains unknown, since microglia appear not to express the TREM2 protein in the human brain, as mentioned above. In the periphery, a significant amount of circulating ApoE is produced by macrophages as well as by the liver. It has been demonstrated that human monocyte-derived macrophages from $\varepsilon 4 / \varepsilon 4$ subjects secret a large amount of ApoE but lack effective cholesterol efflux (Cullen et al., 1998). The $\varepsilon 4 / \varepsilon 4$ macrophages appear to facilitate the development of hypercholesterolemia and to contribute considerably to $\mathrm{AD}$ pathogenesis since hypercholesterolemia is a major risk factor for late-onset $\mathrm{AD}$ (Meng et al., 2014).

\section{CD33 EXPRESSION IN MICROGLIA}

CD33 (also referred to as sialic acid binding immunoglobulinlike lectin-3) used to be considered a myeloid-specific immunomodulatory receptor. Today, it is known that CD33 is also expressed by microglia at both mRNA (Galatro et al., 2017; Gosselin et al., 2017) and protein (Malik et al., 2013) levels in normal human brains. In human $\mathrm{AD}$ brains, the number of CD33-immunoreactive microglia is increased with a positive correlation with the insoluble $\mathrm{A} \beta 42$ burden (Griciuc et al., 2013). $\mathrm{CD} 33$ overexpression associated with the $r s 3865444^{\mathrm{C}}$ risk allele is linked to increased $\mathrm{AD}$ risk, while other single nucleotide polymorphisms (i.e., $r s 3865444^{A}$ and $r s 12459419^{T}$ ) yield nonfunctional $\mathrm{CD} 33$ protein and reduce $\mathrm{AD}$ risk (Bradshaw et al., 2013; Malik et al., 2013). These genetic findings suggest that CD33 mutations cause dichotomous results. Although the protection against $\mathrm{AD}$ onset could be explained by adaptive loss-of-function (Siddiqui et al., 2017), it is still unknown how CD33 mutations contribute to $\mathrm{AD}$ pathogenesis. As a result of the fact that CD33 mutations also change the immunological functions of peripheral monocytes (Bradshaw et al., 2013), the CD33 mutation-altered peripheral immune system may contribute to $\mathrm{AD}$ pathogenesis to some extent, as do altered microglia functions.

\section{DISCUSSION}

As mentioned above, ApoE, TREM2, and CD33 are human GWAS-identified AD risk factors and also DAM-associated genes identified by single-cell RNA analyses on microglia from AD model mice. Based on the cellular expression pattern of the proteins, ApoE, TREM2, and CD33 in humans, the possibility cannot be excluded that functional changes of these molecules due to gene mutations have little to do with microglia in $\mathrm{AD}$ pathogenesis. Instead, the altered functions of ApoE, TREM2, and CD33 may have a close link to peripheral monocytes expressing these proteins and cause a dysregulated immune response, such as chronic systemic inflammation. Furthermore, such functional changes in ApoE, TREM2, and CD33 may indirectly induce the neurotoxic activation of microglia via chronic systemic inflammation, which has been shown to induce neuroinflammation associated with microglial activation (reviewed in Hashioka et al., 2019).

\section{AUTHOR CONTRIBUTIONS}

$\mathrm{SH}$ wrote the manuscript. All authors discussed and edited the manuscript, and read and approved the final manuscript.

\section{FUNDING}

This study was supported by JSPS KAKENHI Grant Number 19K08018 (SH).

\section{ACKNOWLEDGMENTS}

Sincere appreciation is extended to Drs. Patrick and Edith McGeer for their invaluable support. 


\section{REFERENCES}

Atagi, Y., Liu, C. C., Painter, M. M., Chen, X. F., Verbeeck, C., Zheng, $\mathrm{H}$., et al. (2015). Apolipoprotein $\mathrm{E}$ is a ligand for triggering receptor expressed on myeloid cells 2 (TREM2). J. Biol. Chem. 290, 26043-26050. doi: 10.1074/jbc.M115.679043

Bradshaw, E. M., Chibnik, L. B., Keenan, B. T., Ottoboni, L., Raj, T., Tang, A., et al. (2013). CD33 Alzheimer's disease locus: altered monocyte function and amyloid biology. Nat. Neurosci. 16, 848-850. doi: 10.1038/nn.3435

Corder, E. H., Saunders, A. M., Strittmatter, W. J., Schmechel, D. E., Gaskell, P. C., Small, G. W., et al. (1993). Gene dose of apolipoprotein E type 4 allele and the risk of Alzheimer's disease in late onset families. Science 261, 921-923. doi: $10.1126 /$ science. 8346443

Cullen, P., Cignarella, A., Brennhausen, B., Mohr, S., Assmann, G., and Von Eckardstein, A. (1998). Phenotype-dependent differences in apolipoprotein $\mathrm{E}$ metabolism and in cholesterol homeostasis in human monocyte-derived macrophages. J. Clin. Invest. 101, 1670-1677. doi: 10.1172/JCI119887

Fahrenhold, M., Rakic, S., Classey, J., Brayne, C., Ince, P. G., Nicoll, J., et al. (2018). TREM2 expression in the human brain: a marker of monocyte recruitment? Brain Pathol. 28, 595-602. doi: 10.1111/bpa.12564

Galatro, T. F., Holtman, I. R., Lerario, A. M., Vainchtein, I. D., Brouwer, N., Sola, P. R., et al. (2017). Transcriptomic analysis of purified human cortical microglia reveals age-associated changes. Nat. Neurosci. 20, 1162-1171. doi: $10.1038 / \mathrm{nn} .4597$

Gosselin, D., Skola, D., Coufal, N. G., Holtman, I. R., Schlachetzki, J. C. M., Sajti, E., et al. (2017). An environment-dependent transcriptional network specifies human microglia identity. Science 356:eaal3222. doi: 10.1126/science.aal3222

Griciuc, A., Serrano-Pozo, A., Parrado, A. R., Lesinski, A. N., Asselin, C. N., Mullin, K., et al. (2013). Alzheimer's disease risk gene CD33 inhibits microglial uptake of amyloid beta. Neuron 78, 631-643. doi: 10.1016/j.neuron.2013.04.014

Hansen, D. V., Hanson, J. E., and Sheng, M. (2018). Microglia in Alzheimer's disease. J C.ell Biol. 217, 459-472. doi: 10.1083/jcb.201709069

Hashioka, S., Inoue, K., Miyaoka, T., Hayashida, M., Wake, R., Oh-Nishi, A., et al. (2019). The Possible causal link of periodontitis to neuropsychiatric disorders: more than psychosocial mechanisms. Int. J. Mol. Sci. 20:3723. doi: $10.3390 /$ ijms 20153723

Hollingworth, P., Harold, D., Sims, R., Gerrish, A., Lambert, J. C., Carrasquillo, M. M., et al. (2011). Common variants at ABCA7, MS4A6A/MS4A4E, EPHA1, $\mathrm{CD} 33$ and CD2AP are associated with Alzheimer's disease. Nat. Genet. 43, 429-435. doi: $10.1038 / \mathrm{ng} .803$

Jonsson, T., Stefansson, H., Steinberg, S., Jonsdottir, I., Jonsson, P. V., Snaedal, J., et al. (2013). Variant of TREM2 associated with the risk of Alzheimer's disease. N. Engl. J. Med. 368, 107-116. doi: 10.1056/NEJMoa1211103

Kawabori, M., Kacimi, R., Kauppinen, T., Calosing, C., Kim, J. Y., Hsieh, C. L., et al. (2015). Triggering receptor expressed on myeloid cells 2 (TREM2) deficiency attenuates phagocytic activities of microglia and exacerbates ischemic damage in experimental stroke. J. Neurosci. 35, 3384-3396. doi: 10.1523/JNEUROSCI.2620-14.2015

Keren-Shaul, H., Spinrad, A., Weiner, A., Matcovitch-Natan, O., Dvir-Szternfeld, R., Ulland, T. K., et al. (2017). A unique microglia type associated with restricting development of Alzheimer's disease. Cell 169, 1276-1290.e1217. doi: 10.1016/j.cell.2017.05.018

Krasemann, S., Madore, C., Cialic, R., Baufeld, C., Calcagno, N., El Fatimy, R., et al. (2017). The TREM2-APOE pathway drives the transcriptional phenotype of dysfunctional microglia in neurodegenerative diseases. Immunity 47, 566-581.e569. doi: 10.1016/j.immuni.2017.08.008

Lambert, J. C., Heath, S., Even, G., Campion, D., Sleegers, K., Hiltunen, M., et al. (2009). Genome-wide association study identifies variants at CLU and CR1 associated with Alzheimer's disease. Nat. Genet. 41, 1094-1099. doi: $10.1038 /$ ng.439
Lue, L. F., Schmitz, C. T., Serrano, G., Sue, L. I., Beach, T. G., and Walker, D. G. (2015). TREM2 protein expression changes correlate with alzheimer's disease neurodegenerative pathologies in post-mortem temporal cortices. Brain Pathol. 25, 469-480. doi: 10.1111/bpa.12190

Malik, M., Simpson, J. F., Parikh, I., Wilfred, B. R., Fardo, D. W., Nelson, P. T., et al. (2013). CD33 Alzheimer's risk-altering polymorphism, CD33 expression, and exon 2 splicing. J. Neurosci. 33, 13320-13325. doi: 10.1523/JNEUROSCI.1224-13.2013

Mathys, H., Davila-Velderrain, J., Peng, Z., Gao, F., Mohammadi, S., Young, J. Z., et al. (2019). Single-cell transcriptomic analysis of Alzheimer's disease. Nature 570, 332-337. doi: 10.1038/s41586-019-1195-2

Meng, X. F., Yu, J. T., Wang, H. F., Tan, M. S., Wang, C., Tan, C. C., et al. (2014). Midlife vascular risk factors and the risk of Alzheimer's disease: a systematic review and meta-analysis. J. Alzheimers Dis. 42, 1295-1310. doi: 10.3233/JAD-140954

Murakami, M., Ushio, Y., Morino, Y., Ohta, T., and Matsukado, Y. (1988). Immunohistochemical localization of apolipoprotein $\mathrm{E}$ in human glial neoplasms. J. Clin. Invest. 82, 177-188. doi: 10.1172/JCI113568

Park, J. S., Ji, I. J., An, H. J., Kang, M. J., Kang, S. W., Kim, D. H., et al. (2015). Disease-associated mutations of TREM2 alter the processing of N-linked oligosaccharides in the golgi apparatus. Traffic 16, 510-518. doi: $10.1111 /$ tra. 12264

Satoh, J., Kawana, N., Yamamoto, Y., Ishida, T., Saito, Y., and Arima, K. (2013). A survey of TREM2 antibodies reveals neuronal but not microglial staining in formalin-fixed paraffin-embedded postmortem Alzheimer's brain tissues. Alzheimers Res. Ther. 5:30. doi: 10.1186/alzrt184

Siddiqui, S. S., Springer, S. A., Verhagen, A., Sundaramurthy, V., AlissonSilva, F., Jiang, W., et al. (2017). The Alzheimer's disease-protective CD33 splice variant mediates adaptive loss of function via diversion to an intracellular pool. J. Biol. Chem. 292, 15312-15320. doi: 10.1074/jbc.M117.7 99346

Suarez-Calvet, M., Morenas-Rodriguez, E., Kleinberger, G., Schlepckow, K., Araque Caballero, M. A., Franzmeier, N., et al. (2019). Early increase of CSF sTREM2 in Alzheimer's disease is associated with tau relatedneurodegeneration but not with amyloid-beta pathology. Mol. Neurodegener. 14:1. doi: 10.1186/s13024-018-0301-5

Thrash, J. C., Torbett, B. E., and Carson, M. J. (2009). Developmental regulation of TREM2 and DAP12 expression in the murine CNS: implications for Nasu-Hakola disease. Neurochem. Res. 34, 38-45. doi: 10.1007/s11064-0089657-1

Uchihara, T., Duyckaerts, C., He, Y., Kobayashi, K., Seilhean, D., Amouyel, P., et al. (1995). ApoE immunoreactivity and microglial cells in Alzheimer's disease brain. Neurosci. Lett. 195, 5-8. doi: 10.1016/0304-3940(95)1 $1763-\mathrm{M}$

Villegas-Llerena, C., Phillips, A., Garcia-Reitboeck, P., Hardy, J., and Pocock, J. M. (2016). Microglial genes regulating neuroinflammation in the progression of Alzheimer's disease. Curr. Opin. Neurobiol. 36, 74-81. doi: 10.1016/j.conb.2015.10.004

Conflict of Interest: The authors declare that the research was conducted in the absence of any commercial or financial relationships that could be construed as a potential conflict of interest.

Copyright $\odot 2020$ Hashioka, Inoue, Takeshita and Inagaki. This is an open-access article distributed under the terms of the Creative Commons Attribution License (CC $B Y)$. The use, distribution or reproduction in other forums is permitted, provided the original author(s) and the copyright owner(s) are credited and that the original publication in this journal is cited, in accordance with accepted academic practice. No use, distribution or reproduction is permitted which does not comply with these terms. 